\title{
Characterization of virgin avocado oil obtained via advanced green technique
}

\begin{abstract}
The quality characteristics, bioactive phytochemicals, volatile compounds, and antioxidant capacities of virgin avocado oil extracted using a couple of green methods, namely, subcritical $\mathrm{CO} 2$ extraction (SCO2) and ultrasound-assisted aqueous extraction (UAAE), are compared with the oil extracted using the conventional solvent extraction. Results indicate the quality properties of avocado oil are unaffected by extraction methods. The total phenolic content of avocado oil is in the range of $111.27-130.17 \mathrm{mg}$ GAE$/ 100 \mathrm{~g}$ and the major phytosterol is $\beta$ sitosterol (1.91-2.47 $\mathrm{g} \mathrm{kg}-1$ ). Avocado oil extracted using SCO2 exhibits two to four times greater levels of $\alpha$ - and $\gamma$-tocopherols than solvent extraction and UAAE. The volatile components associated with nutty and grassy flavors are only detected in avocado oil extracted under low-temperature extraction conditions such as SCO2 and UAAE. Based on the antioxidant capacity tests, avocado oil obtained by SCO2 exhibits the strongest antioxidant capacity compared with solvent extraction and UAAE.
\end{abstract}

Keyword: Antioxidant capacity; Avocado oil; Bioactive phytochemicals; Subcritical CO2 extraction; Ultrasound-assisted aqueous extraction 